NASA Technical Memorandum 105584

\title{
Long Time Behavior of Unsteady Flow Computations
}

S.I. Hariharan

University of Akron

Akron, Ohio.

and Institute for Computational Mechanics in Propulsion

Lewis Research Center

Cleveland, Ohio

March 1992

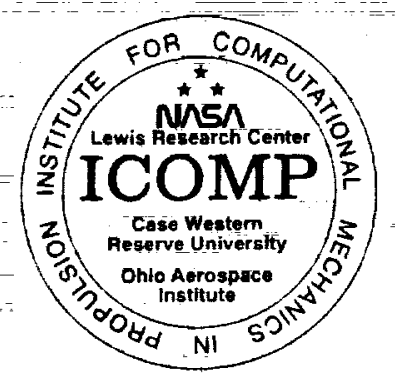




\title{
Long Time Behavior of Unsteady Flow Computations
}

\author{
S.I. Hariharan \\ University of Akron \\ Department of Mathematical Sciences \\ Akron, Ohio 44325 \\ and Institute for Computational Mechanics in Propulsion \\ Lewis Research Center \\ Cleveland, Ohio 44135
}

\begin{abstract}
This paper addresses a specific issue of time accuracy in the calculations of external aerodynamic problems. The class of problems that is discussed here consists of inviscid compressible subsonic flows. These problems are inherently governed by a convective equation. This is readily seen by linearizing the Euler equation which results in a convective wave equation for the pressure. A key mathematical issue that is not well understood in literature for these problems is the long time behavior of the solution. This is an important aspect if one desires transient calculations of problems governed by the Euler equations or its derivatives such as the small disturbance equations or the potential formulations of the gust problem. In particular, difficulties arise for two-dimensional problems. In two dimensions the time decay rate of solutions of the wave equation is known to be rather slow. This applies to the convective wave equation as well. The consequences are rather severe if one focuses on the time accuracy of solutions of problems governed by the Euler equations.

In concert with the above mentioned problem, exterior flows require proper modeling of boundary conditions. In particular, computations of these flow problems require truncation of infinite regions into finite regions with the aid of artificial boundaries. On these boundaries one must impose boundary conditions that are consistent with the physics as well as guarantee consistency with the original problem posed in the unbounded region. Moreover, these boundary conditions must have accuracy in time as well as space. Some of the well-known procedures do address the issues of spatial accuracy and have remedy for these conditions. Unfortunately, these procedures do not address the time accuracies, which are crucial for the transient problems. Our treatment is discussed in detail and examples are presented to verify the results.
\end{abstract}

\footnotetext{
'Research for the author was supported by the National Science Foundation, DMS-8921189 and by ICOMP, NASA Lewis Research Center (work funded under NASA Cooperative Agreement NCC3-233).
} 


\section{Introduction}

This paper deals with unanswered aspects of long time behavior of unsteady flow computations in two dimensions. Many of the available codes such as the FLO series deal with steady flows and their accuracies are well tested. State of the art grid generations and even complicated geometrical structures are included in these codes. While these developments reflect the maturity of computational fluid dynamics, the goal of the field is to understand aerodynamics in its entirety which includes the unsteady phenomena. It is important to understand this phenomena if one is to achieve successful calculations on biproducts such as far field acoustics. A common tendency is to use steady fluid dynamics codes for unsteady calculations. Such calculations yield either unsatisfactory resolution of time accuracies or break down in finite time. The classical problem of subsonic-transonic compressible flows past a cylinder is an unresolved issue for this very reason. For example one can see a documentation of such computations in [15]. The key reason why this is difficult is due to lack of understanding in the modeling of far field boundary conditions that naturally plays an important if not an essential role in these computations.

It is an open issue whether one can predict solutions of unsteady Euler or Navier-Stokes equations in a satisfactory manner. A recent paper by [2] deals with numerical computations of viscous flows past a circular cylinder. The modeling of required far field approximate pressure condition was obtained using a theory proposed by [1] which is accurate for high frequencies. This limits the solutions to be accurate for short times. They also proposed approximate boundary conditions using characteristic variables which works better than the above said pressure boundary condition. It is not clear if these conditions mantain time accuracy of the solution and it needs to be addressed. This concern forms the basis of this paper. It should be emphasized the workers realized similar difficulties ahead of time and concentrated on linearized Euler equations and in the frequency domain. Namely, the work of Goldstein [12] stands out in the literature. Corrections to this model were proposed by Atassi [13] and subsequently he and his coworkers (e.g. see [14]) succeeded in predicting unsteady aerodynamic calculations through numerical procedures. These formulations and calculations are done through potential methods and the resulting equations are nonconstant coefficient second order linear partial differential equations. Calculations are accurate within the linearized limits. In certain cases, such as flat plate air foils, the theory behind these equations is well understood. As a result, these potential 
formulations have firm foundations. Recently several authors are beginning to solve linearized Euler equations directly. It is not clear if there are any real advantages to these procedures. It appears that the mathematical foundations do need careful work. Moreover, associated far field boundary conditions consistent with the linearized equations require a careful study. This is the other aspect this paper addresses.

Two-dimensional problems governed by any wave equation, unlike their one- or threedimensional counterparts, suffer severe slow decay rates under certain conditions. Whether the problem is posed in the frequency domain or in the time domain, the problem of slow decay rates occurs. For example, for a very low frequency problem it would appear from a conceptual point of view that the terms multiplied by the frequencies could be dropped to consider a problem in the absence of those corresponding terms. A typical situation is the problem governed by the Helmholtz equation, where reduction is the Laplace equation. Similar arguments hold for the time dependent wave equation. If in the long time there is a steady state, the analogous situation is to consider the steady state part. These discussions imply that there is interplay between low frequencies and long time behavior. The researchers who looked at these questions considered only isolated issues. In this paper an attempt has been made to connect the theoretical issues behind these problems to aerodynamic problems.

In section two we discuss the behavior of the solution of reduced wave equations in exterior regions. In this section we point out the potential problems in considering the zero frequency limit or the steady state problems. Moreover, we show the proper form of the boundary operators one should consider in the prescription of radiation boundary conditions. In section three we consider a true time dependent model in which the corresponding corrections to the radiation boundary operator are discussed. Here we include available well-known procedures that are commonly used in the computational fluid dynamic literature to show these corrections in perspective. Numerical solutions are shown to establish the validity of the theory.

Finally in section four we provide extension of the theory proposed in section 3 for the treatment in the far field. This treatment should hold for the Euler equations and their linearized versions. The corresponding numerical treatment will be discussed elsewhere.

\section{Low frequency behavior of the reduced wave equation}

Here we show a potential problem that arises with the reduced wave equation. In particular we shall discuss the low frequency behavior that arises from problems with Dirichlet boundary 
conditions. The reason for discussing such behavior is to establish the slow decay rates of the two dimensional wave equation. These decay rates play an important role in the construction of approximate boundary operators that are vital to the constructions of solutions. At the outset we want to emphasize that these decay rates and associated problems only apply to the two-dimensional wave and wave-like equations.

We shall begin with the two-dimensional problem that will motivate the decay rates in question. We consider a classical soft obstacle problem in two dimensions. In particular we consider the following boundary value problem $\left(P_{k}\right)$ :

$$
\begin{aligned}
\Delta u+k^{2} u & =0 & & \text { in } \Omega_{\mathrm{e}} \subset \mathcal{R}^{2} \\
u & =g_{k} & & \text { on } \Gamma \\
\lim _{r \rightarrow \infty}\left(u_{r}-i k u\right) & =0 & & r=\sqrt{x^{2}+y^{2}}
\end{aligned}
$$

where $\Omega_{e}$ is the exterior to a bounded region $\Omega$ and $\Gamma$ is its boundary. For the soft obstacle problem $g_{k}=-u_{i n c}$ where $u_{i n c}$ is the incident field.

The key question we address is what happens to the problem as $k \rightarrow 0$ ? To discuss this issue we consider an appropriate zero frequency problem. This problem is constructed in such a way that it preserves the zero frequency behavior on the equation and the boundary condition on $\Gamma$. However, the radiation condition at infinity is replaced by a boundedness condition at infinity and is as follows:

$$
\begin{aligned}
\Delta v & =0 & & \text { in } \Omega_{\mathrm{e}} \subset \mathcal{R}^{2} \\
v & =g_{0} & & \text { on } \Gamma \\
|v| & \leq M & & \text { or } \mathrm{v} \rightarrow \mathrm{A} \log |\overrightarrow{\mathbf{x}}| \text { as }|\overrightarrow{\mathbf{x}}|=\mathbf{r} \rightarrow \infty .
\end{aligned}
$$

We call this problem to be $\left(P_{0}\right)$. It has been shown in the literature [10], [8] that in the limit as $k \rightarrow 0$ the solution of $\left(P_{k}\right)$ approaches the solution of $\left(P_{0}\right)$. However, this limit process is a slow one and we want to make a case for it as it has everything to do with constructions of boundary conditions for numerical simulations. Before we introduce the results let us focus our attention on the structure of $v_{\text {inc }}=-g_{0}$. The choice of $v_{\text {inc }}$ depends on $u_{\text {inc }}$. Examples are as follows:

a) $u_{\text {inc }}=e^{i k x} \quad$ (plane wave)

Then the corresponding zero frequency limit is readily seen and is $v_{\text {inc }}=1$ 
b) $\quad u_{\text {inc }}=-\frac{i}{4} H_{0}^{(1)}\left(k\left|\vec{x}-\vec{x}_{0}\right|\right) \quad$ (singular source)

Then the corresponding zero frequency limit is taken as the principal leading spatial singularity $v_{\text {inc }}=\frac{1}{2 \pi} \log \left|\vec{x}-\vec{x}_{0}\right|$. A discussion of this choice is seen in [7] and is motivated by several issues including an obvious one: the asymptotic behavior of the Hankel function for small arguments. Now we state some of the key results:

a) (Crude) : $u \rightarrow v$ as $k \rightarrow 0$

b) (Sharp): $u=v+O\left(\frac{1}{\ln k}\right)$

c) (Sharper): $\quad u=v+\frac{c f_{0}}{\beta-c_{0}}+O\left(k^{2}(\ln k)^{2}\right)$

The results in a) are due to an old work of MacCamy [8]. The results in b) are later observed by many others, namely Muravei [10], Werner [16] and Kress [17] which indicate the slow decay rates. The result $c$ ) is due to the author and his coworkers and the results appear in $[9],[6]$ and [7]. To motivate the implication of these results first let us demonstrate the effect of these decays numerically. For this purpose we have taken a plane wave incident with unit amplitude. It is easily seen from problem $\left(P_{0}\right)$ that the solution is $v_{\mathrm{s}}=-1$ and the total field $v=v_{\text {inc }}+v_{s}=0$. This solution is compared with the total solution for the nonzero frequency problem $\left(P_{k}\right)$ with $u=u_{\text {inc }}+u_{s}$. Figure 1 shows magnitude of $u$ for the frequencies $10^{-2}$, $10^{-3}$ and $10^{-4}$ respectively. Clearly one can see that even for these very low frequencies the solution does not approach the correct low frequency limit of zero rapidly. The frequency or the wave number approaching zero corresponds to the long time limit of the solution to the corresponding time dependent wave propagation problem. In particular for moderate times or frequency one cannot expect to use any properties of the steady state problem and in this case the solution of the zero frequency problem $\left(P_{0}\right)$. This message has serious impacts on unsteady flow computations.

In light of the above discussion, we would like to outline a correction to this zero frequency computation. These ideas will later be transferred to an appropriate time domain problem and at the end we shall propose ideas as to how to extend the theory to the Euler equations. In particular we outline a theory that appears in [6], [7] to show the sharp result b) and the sharper result c). The construction of these results involve potential theory. We use potential theoretic representation for the solution. Specifically we use single layer potential representations. Let 
$u(\vec{x})$ be the total field in $\left(P_{k}\right)$, i.e.,

$$
u(\vec{x})=u_{i n c}(\vec{x})+u_{s}(\vec{x})
$$

Then, for the problem $\left(P_{k}\right)$ the representation is as follows:

$$
u(\vec{x})=\int_{\Gamma} \sigma(\vec{y}) G_{k}(\vec{x}, \vec{y}) d s_{\vec{y}}+u_{i n c}(\vec{x}), \quad \vec{x} \in \Omega_{e}
$$

where

$$
G_{k}(\vec{x}, \vec{y})=\frac{-i}{4} H_{0}^{(1)}\left(k\left|\vec{x}-\vec{x}_{0}\right|\right)
$$

is the free space Green's function. The scattered field satisfies the Sommerfeld's radiation condition due to this Green's function. One obtains a boundary integral equation for the determination of $\sigma(\vec{y})$ that is defined on the boundary $\Gamma$ using the boundary condition on $\Gamma$.

$$
\int_{\Gamma} \sigma(\vec{y}) G_{k}(\vec{x}, \vec{y}) d s_{\vec{y}}+u_{i n c}(\vec{x})=0, \vec{x} \in \Gamma
$$

A similar construction with the free space Green's function for the Laplace's equation is made for the problem $P_{0}$ noting that any representation of the solution is subject to an arbitrary constant.

$$
v(\vec{x})=\int_{\Gamma} \mu(\vec{y}) G_{0}(\vec{x}, \vec{y}) d s_{\vec{y}}+v_{i n c}(\vec{x})+c, \vec{x} \in \Omega_{e}
$$

with

$$
\int_{\Gamma} \mu(\vec{y}) d s_{\vec{y}}=-1
$$

where

$$
G_{0}(\vec{x}, \vec{y})=\frac{1}{2 \pi}(\log |\vec{x}-\vec{y}|) .
$$

The condition described by equation (8) takes care of the arbitrariness of the constant $c$ and the boundedness of the solution at infinity. Again demanding the boundary condition on $\Gamma$ we arrive at the boundary integral equation

$$
\int_{\Gamma} \mu(\vec{y}) G_{0}(\vec{x}, \vec{y}) d s_{\vec{y}}+v_{\text {inc }}(\vec{x})+c=0, \vec{x} \in \Gamma .
$$

Thus the complete solution of $\left(P_{0}\right)$ is determined through solving the equations of the pair of equations (8) and (10) simultaneously. The key idea of the low frequency correction relies on relating $\sigma$ and $\mu$ with appropriate low frequency corrections. Several of the quantities that arise can be related to the asymptotic formula for small values of $z$

$$
\frac{-i}{4} H_{0}^{(1)}(z)=\frac{1}{2 \pi}\left(\log \left(\frac{z}{2}\right)+\gamma\right)-\frac{i}{4}+O\left(z^{2} \ln z\right)
$$


where

$$
\gamma=.5772156649 \cdots
$$

is the Euler constant. Using this expansion in equation (6) we obtain a related integral equation in the following form

$$
\int_{\Gamma} \tilde{\sigma}(\vec{y}) G_{0}(\vec{x}, \vec{y}) d s_{\vec{y}}+\beta\left(\int_{\Gamma} \tilde{\sigma}(\vec{y}) d s_{\vec{y}}+1\right)+v_{i n c}(\vec{x})=0
$$

where

$$
\beta=\frac{1}{2 \pi}\left(\log \left(\frac{k}{2}\right)+\gamma\right)-\frac{i}{4} .
$$

Successive approximation by considering equation (4) and (13) yields ([6])

$$
\sigma=\tilde{\sigma}+O\left(k^{2} \ln k\right)
$$

Further it has been shown in [6] that

$$
\tilde{\sigma}(\vec{x})=\mu(\vec{x})+\frac{c f_{0}(\vec{x})}{\beta-c_{0}}, \vec{x} \in \Gamma
$$

where $f_{0}(\vec{x})$ and the real constant $c_{0}$ satisfy the following equations :

$$
\begin{gathered}
\int_{\Gamma} f_{0}(\vec{y}) G_{0}(\vec{x}, \vec{y}) d s_{\vec{y}}+c_{0}=0 \\
\int_{\Gamma} f_{0}(\vec{y}) d s_{\vec{y}}=1 .
\end{gathered}
$$

Use of this result leads to the results in c). In particular two low frequency corrections can be constructed. One is for $k|\vec{x}|$ small (the results in [6]) and another one that is proposed in the recent paper [7] for $k$ small globally. The emphasis of the latter corrections are to include the low frequency corrections both in the near field as well as the far field. Those corrections are as follows

$$
\tilde{w}(\vec{x})=\int_{\Gamma} \tilde{\sigma}(\vec{y}) G_{0}(\vec{x}, \vec{y}) d s_{\vec{y}}+\beta\left(\int_{\Gamma} \tilde{\sigma}(\vec{y}) d s_{\vec{y}}+1\right)+v_{\text {inc }}(\vec{x})
$$

which is the one that appears in [6].

$$
w(\vec{x})=\int_{\Gamma} \tilde{\sigma}(\vec{y}) G_{k}(\vec{x}, \vec{y}) d s_{\vec{y}}+u_{i n c}(\vec{x})
$$

This is the improved result that is valid both in the near field and in the far field. To illustrate the effectiveness of these approximations we solved for $u$, the full solution of Helmholtz equation, 
and the approximations $\tilde{w}$ and $w$ for the case of a circular obstacle and a plane wave. All the results were calculated numerically. Figures 2 and 3 show the effect of these corrections as predicted by the theory for reduced frequencies $k=10^{-2}$ and $k=10^{-4}$ respectively.

The important information that is related to the computations of exterior aerodynamic calculations is as follows: Suppose one wants to compute solutions within a truncated region with the aid of an artificial boundary. Then for the well-posedness of the problem within the truncated region one must have normal derivatives prescribed on the boundary in an appropriate form. For example one can realize this form by considering the Sommerfeld's radiation condition. The operator that we propose is

$$
\frac{\partial u}{\partial n}(\vec{x})=\frac{\partial v}{\partial n}(\vec{x})+\frac{1}{\beta-c_{0}} \int_{\Gamma} f_{0}(\vec{y}) \frac{\partial G_{0}}{\partial n} d s_{\vec{y}}+\mathcal{O}\left(k^{2}(\log k)^{2}\right)
$$

A similar relation holds if one is interested in specifying the normal derivative of the scattered field only. In this case $u$ will be replaced by $u_{s}$ and $v$ will be replaced by $v_{s}$. The importance of this condition is that in the low frequency limits it is important to correct the zero frequency operator with the logarithmic terms which play a significant role. This will be evident in the next section where we shall deal with a true time dependent situation and the structure of these logarithmic corrections in the frequency translates to logarithmic corrections in time in the artificial boundary operators. These constructions were made from a computational point of view but are consistent with the abstract theoretical results posed in [10].

A point of comfort and warning is that these corrections are not necessary in the case of a hard obstacle. That is, the Dirichlet condition on $\Gamma$ is replaced with a Neumann condition

$\frac{\partial u_{e}}{\partial n}=-\frac{\partial u_{i n c}}{\partial n}$. It can be shown that the decay rates are much faster for this condition and does not involve any logarithmic corrections. Potential formulations that were introduced by Goldstein [12] and Atassi [13] fall into this category. As a result the computational results presented in [14] do behave well in the low frequency limits. However, issues dealing with the time dependent problems, in particular problems governed by the Euler equations, are in need of further theoretical backing. We show a preliminary study in the next section.

\section{Implications to a True Time Dependent Model}

In this section we motivate a time dependent model problem that is used to indicate potential troubles in two dimensions. Specifically, we shall focus our attention on construction of absorbing boundary conditions on artificial boundaries. The model problem that we propose is 
governed by the wave equation exterior to a circle of radius $r_{0}$ with a time dependent data on $r_{0}$ which asymptotes to a constant value as $t \rightarrow \infty$. For computational purposes we truncate the infinite region by an artificial boundary at $r=R$. At this boundary there is an exact condition (in the mathematical sense) that takes care of the effects of extending the region to infinity. The model problem is as follows:

$$
\begin{array}{cc}
u_{t t}=u_{r r}+\frac{1}{r} u_{r}, & r_{0}<r<R \\
u\left(r_{0}, t\right)=1+g(t) & \text { at } r=R . \\
u_{r}=\mathcal{F}(u) & r_{0}<r<R \\
u(r, 0)=u_{t}(r, 0)=0 & \text { as } t \rightarrow \infty
\end{array}
$$

This model is designed to have the long time behavior of a solution $u \rightarrow 0$ as $t \rightarrow \infty$. The operator $\mathcal{F}(u)$ is the exact operator known only theoretically. It has a well defined structure in the transformed domain which we discuss now. The initial values suggest that it is easier to work with the Laplace transforms. Doing so one obtains

$$
\begin{gathered}
s^{2} \bar{u}=\bar{u}_{r r}+\frac{1}{r} \bar{u}_{r}, \quad r_{0}<r<R \\
\bar{u}(r ; s)=\bar{g} \\
\frac{\partial \bar{u}}{\partial r}(R ; s)=\overline{\mathcal{F}}(\bar{u})
\end{gathered}
$$

The operator $\overline{\mathcal{F}}$ in the transformed space is readily verified by considering the solution of equation (2.27) in the exterior region and is as follows:

$$
\overline{\mathcal{F}}(\bar{u})=\frac{s K_{0}^{\prime}(s R)}{K_{0}(s R)} \bar{u}
$$

Available well-known boundary condition procedures rely on obtaining approximations of the symbol or the ratios of the modified Bessel fucnctions. Here we provide some of these approximations and operators. Let $\bar{B}$ be the approximation of $\mathcal{F}$. Then

1. Engquist and Halpern [11]

$$
\bar{B}=-s
$$


which is the crudest approximation of $\overline{\mathcal{F}}$. Then the approximate boundary operator in the transformed space is

$$
\frac{\partial \bar{u}}{\partial r} \approx \bar{B}(\bar{u})
$$

Inversion of the transform results in a time domain operator

$$
\frac{\partial u}{\partial r}+\frac{\partial u}{\partial t}=0
$$

The important part to consider here is the error in the approximation (2.31) using asymptotic properties of the modified Bessel functions for small and large arguments. This in turn will suggest the order of the error in real time. Doing so we obtain

$$
\overline{\mathcal{F}}-\bar{B}= \begin{cases}\frac{1}{2 \bar{R}} & \text { as } \mathrm{s} \rightarrow \infty \\ O\left(\frac{1}{\ln s}\right) & \text { as } \mathrm{s} \rightarrow 0\end{cases}
$$

2. Bayliss and Turkel [4] first order

Analogous list in this are boundary operators in the transform domain

$$
\bar{B}=-s-\frac{1}{2 R}
$$

the boundary operator in the time domain

$$
\frac{\partial u}{\partial r}+\frac{\partial u}{\partial t}+\frac{1}{2 R} u=0
$$

and the errors behave like

$$
\overline{\mathcal{F}}-\bar{B}=\left\{\begin{array}{l}
O\left(\frac{1}{s}\right) \text { as } \mathrm{s} \rightarrow \infty \\
-\frac{1}{2 R} \text { as } \mathrm{s} \rightarrow 0
\end{array}\right.
$$

3. Bayliss and Turkel [4] second order

Here the boundary operator in the transform domain is

$$
\bar{B}=-\frac{s^{2}+\frac{3 s}{2 R}+\frac{3}{8 R^{2}}}{s+\frac{1}{R}}
$$

and its translation in the time domain is

$$
\left(\frac{\partial}{\partial r}+\frac{\partial}{\partial t}+\frac{5}{2 R}\right)\left(\frac{\partial}{\partial r}+\frac{\partial}{\partial t}+\frac{1}{2 R}\right) u=0
$$

The errors in this case are

$$
\overline{\mathcal{F}}-\bar{B}= \begin{cases}O\left(\frac{1}{s^{2}}\right) & \text { as } \mathrm{s} \rightarrow \infty \\ -\frac{3}{8 R} & \text { as } \mathrm{s} \rightarrow 0\end{cases}
$$


4. Hagstrom [5] (part of current study)

The boundary operator in the transform domain is

$$
\bar{B}=-\frac{s\left(s+\frac{1}{2 R}+\delta\right)}{s+\delta}
$$

and its translation in the time domain is

$$
\left(\frac{\partial}{\partial t}+\delta\right)\left(\frac{\partial u}{\partial r}\right)+\left(\frac{\partial}{\partial t}+\frac{1}{2 R}+\delta\right) \frac{\partial u}{\partial t}=0
$$

Finally the errors behave like

$$
\overline{\mathcal{F}}-\bar{B}= \begin{cases}O\left(\frac{1}{a}\right) & \text { as } \mathrm{s} \rightarrow \infty \\ O\left(\frac{1}{\ln s}\right) & \text { as } \mathrm{s} \rightarrow 0\end{cases}
$$

We propose to correct the errors in the low frequency limit, i.e., the long time behavior in constructions of these operators. This is the punch line of this paper and again we emphasize that this idea is crucial if one were to compute directly time dependent problems. For this purpose let us observe that if one were to include the next order correction to the asymptotics for small $s$, we obtain the following properties:

$$
\bar{u}_{r} \sim \begin{cases}-\left(s+\frac{1}{2 R}\right) \bar{u} & \text { as } s \rightarrow \infty \\ -\frac{1}{R \ln \left(\operatorname{Re}^{\tau} \frac{\frac{1}{2}}{2}\right)} \bar{u} & \text { as } s \rightarrow 0\end{cases}
$$

where $\gamma$ is the Euler's constant. Now consider the case where

$$
u \rightarrow 1 \quad \text { as } t \rightarrow \infty
$$

which has the behavior in the transform domain

$$
\bar{u} \rightarrow \frac{1}{s} \quad \text { as } s \rightarrow 0
$$

In particular

$$
\bar{u}_{r} \sim-\frac{1}{R s \ln \left(R e^{\gamma} \frac{s}{2}\right)} \quad \text { as } s \rightarrow 0 .
$$

To obtain the corrected behavior in the time domain we look for a function $G(t)$ so that

$$
\mathcal{L}\{G(t) u\}=-\frac{1}{R s \ln \left(R e^{\gamma} \frac{1}{2}\right)} \quad \text { as } s \rightarrow 0 .
$$


Construction of such a function will be found in [3]. The key result here is Proposition:

$$
G(t)=\frac{1}{R\left[\ln \left(\frac{2 t}{R}\right)+D\right]}
$$

where $D$ is a constant such that $D>e^{2}$. The proposed boundary operator here has the form

$$
\left(\frac{\partial}{\partial t}+\delta\right) \frac{\partial u}{\partial r}=-\left(\frac{\partial}{\partial t}+\frac{1}{2 R}+\delta\right) \frac{\partial u}{\partial t}-\delta G(t) u \text {. }
$$

We compare this operator with the operator in category 4 . When $\delta$ is a constant and $G=0$ one retrieves this operator. The function $\delta(t)$ is determined by matching the next asymptotic correction in space (i.e., $O\left(\frac{1}{R^{3}}\right)$ ). This yields

$$
\delta=\frac{1}{4 R} \frac{1}{1-2 R G} .
$$

The details of these calculations will be found in [3].

We demonstrate the effect of this proposition through a numerical simulation. For this simulation a specific choice of $g(t)$ is considered. It is modeled in such a way that it has oscillatory decay. The form is

$$
u\left(r_{0}, t\right)=1-\frac{\cos 2 \pi t}{1+t^{2}} \quad t>0 .
$$

Moreover, this problem does not have an explicit analytic solution. Therefore, the numerical simulation and comparison is made by doing the calculation in a large domain $r_{0}<r<R$ with $r_{0}=1$ and $R=252$. The value of $R$ is chosen in such a way that the waves do not hit this artificial boundary so that no reflection in the computational domain is introduced in the time period (500 nondimensional units). The specific scheme that is used for computation is an explicit second order scheme (both in time and space). The graphs and associated results are given in Figure 4. In addition the results that are plotted in the figure represent the magnitude of error in time at a fixed point within the computational domain. This point in this case is chosen to be $r=2$. The results are consistent with the predicted theory. In particular the theory predicts that the results of Engquist and Halpern [11] have the largest error for short times and is demonstrated in the graph. However, for large times this error tends to decay but only at a very short rate which is consistent with the key emphasis of this paper. The second and third graphs are due to the computations using the Bayliss and Turkel [4] boundary operators of orders one and two respectively. Clearly one sees smaller errors at short times and the errors 
have a tendency of growing larger (they do indeed though not shown here). Finally the last curve is the current proposal [3] and has errors less than a percent for all times and it behaves well for short, long and in between times. These constructions are a guide to an abbreviated proposal that is given for aerodynamic problems, specifically for the Euler equations.

\section{Extensions to Aerodynamics}

Here we extend the ideas presented in the earlier sections to aerodynamic problems governed by the Euler equations. A similar analysis can be done for the viscous flows governed by the Navier-Stokes equations which is not presented here. It should be noted that to extend the analysis of the model problem it is vital to have a linear problem. Clearly the Euler equations or the Navier-Stokes equations are not linear. However, the analysis which we seek is for the far field boundary operators and is reasonable to assume linearity of the equations there. The form of the Euler equations we use here is as follows:

$$
\begin{gathered}
\frac{\partial \rho}{\partial t}+\operatorname{div}(\rho \underline{u})=0 \\
\rho \frac{D \underline{u}}{D t}=-\nabla p \\
\rho \frac{D e}{D t}+p \operatorname{div} \underline{u}=0
\end{gathered}
$$

There are a variety of ways to linearize these equations about a state. In this case we consider the state at infinity. Since we are dealing with a system of first order equations, additional boundary conditions are required according to the hyperbolic theory. The treatments that are presented here are for subsonic inflow as well as outflow. Along these lines Gustafsson [18] proposed a linearization with the variables $q=\operatorname{div} \underline{u}$, the divergence of the velocity field, and the entropy $S=\ln \left(\frac{p}{\rho^{\gamma}}\right)$. Noting that $\underline{\omega}=$ curl $\underline{u}$ the linearized equations look like

$$
\begin{gathered}
\frac{\partial S}{\partial t}+u_{\infty} \frac{\partial S}{\partial x}=0 \\
\frac{\partial \omega}{\partial t}+u_{\infty} \frac{\partial \omega}{\partial x}=0 \\
\left\{\begin{array}{l}
\frac{\partial q}{\partial t}+u_{\infty} \frac{\partial q}{\partial x}+\rho_{\infty} \Delta p=0 \\
\frac{\partial p}{\partial t}+u_{\infty} \frac{\partial p}{\partial x}+\rho_{\infty} c_{\infty}^{2} q=0
\end{array}\right.
\end{gathered}
$$


In the last two equations, elimination of $q$ yields a convective wave equation for the pressure

$$
\left(\frac{\partial}{\partial t}+u_{\infty} \frac{\partial}{\partial x}\right)^{2} p=c_{\infty}^{2} \Delta p
$$

This is the place where the connection to our earlier analysis holds. The treatment of boundary conditions for the pressure is subject to the structure of the solutions of this equation. Before we proceed to discuss these equations we point out that the linearized equations for the entropy and vorticity suggest that at points of inflow one must specify $S$ and $\omega$. These are dictated by the hyperbolic theory as well.

Now we set up an appropriate initial boundary value problem that will be used to extend the analysis in the previous section. The model to be considered here is:

$$
\begin{gathered}
\psi_{t t}+2 U_{\infty} \psi_{x t}+U_{\infty}^{2} \psi_{x x}=C_{\infty}^{2} \psi_{x x}+C_{\infty}^{2} \psi_{y y} \\
\psi=\psi_{t}=0 \text { at } t=0
\end{gathered}
$$

where $\psi=p-p_{\infty}$. Taking the Laplace transform with respect to $t$ yields:

$$
s^{2} \bar{\psi}+2 U_{\infty} s \bar{\psi}_{x}+U_{\infty}^{2} \bar{\psi}_{x x}=C_{\infty}^{2} \bar{\psi}_{x x}+C_{\infty}^{2} \bar{\psi}_{y y}
$$

Now we make a dependent variable change from $\bar{\psi}$ to $\phi$ in the form

$$
\bar{\psi}=e^{\lambda x} \phi
$$

This yields the following calculations:

$$
\begin{gathered}
\bar{\psi}_{x}=\lambda e^{\lambda x} \phi+e^{\lambda x} \phi_{x} \\
\bar{\psi}_{x x}=\lambda^{2} e^{\lambda x} \phi+2 \lambda e^{\lambda x} \phi_{x}+e^{\lambda x} \phi_{x x} \\
\bar{\psi}_{y y}=e^{\lambda x} \phi_{y y}
\end{gathered}
$$

Substituting (64-66) in the transformed equation we obtain

$$
s^{2} \phi+2 U_{\infty} s \lambda \phi+2 U_{\infty} s \phi_{x}=\left(C_{\infty}^{2}-U_{\infty}^{2}\right)\left(\phi_{x x}+2 \lambda \phi_{x}+\lambda^{2} \phi\right)+C_{\infty}^{2} \phi_{y y} .
$$

We pick the free parameter $\lambda$ (to eliminate the first derivative term) so that

$$
\lambda=\frac{U_{\infty} s}{C_{\infty}^{2}-U_{\infty}^{2}} .
$$


The remaining terms involving $\lambda$ yield

$$
\begin{gathered}
s^{2}+2 U_{\infty} s \lambda-\left(C_{\infty}^{2}-U_{\infty}^{2}\right) \lambda^{2}=s^{2}+\frac{2 U_{\infty}^{2} s^{2}}{C_{\infty}^{2}-U_{\infty}^{2}}-\frac{U_{\infty}^{2} s^{2}}{C_{\infty}^{2}-U_{\infty}^{2}} \\
=s^{2}+\frac{U_{\infty}^{2} s^{2}}{C_{\infty}^{2}-U_{\infty}^{2}}=\frac{s^{2} C_{\infty}^{2}}{C_{\infty}^{2}-U_{\infty}^{2}} .
\end{gathered}
$$

Thus the equation for $\phi$ becomes

$$
\frac{s^{2} C_{\infty}^{2}}{C_{\infty}^{2}-U_{\infty}^{2}} \phi=\left(C_{\infty}^{2}-U_{\infty}^{2}\right) \phi_{x x}+C_{\infty}^{2} \phi_{y y}
$$

or

$$
\frac{s^{2}}{C_{\infty}^{2}-U_{\infty}^{2}} \phi=\left(1-M_{\infty}^{2}\right) \phi_{x x}+\phi_{y y} .
$$

Let

$$
\begin{gathered}
\tilde{s}=\frac{s}{\sqrt{C_{\infty}^{2}-U_{\infty}^{2}}} \\
\tilde{x}=\frac{x}{\sqrt{1-M_{\infty}^{2}}} \\
\tilde{y}=y .
\end{gathered}
$$

Then the equation for $\phi$ becomes

$$
\phi_{\tilde{x} \tilde{x}}+\phi_{\tilde{y} \tilde{y}}=\tilde{s}^{2} \phi .
$$

Radially symmetric solutions satisfy

$$
\phi_{\tilde{f}}=\tilde{s} \frac{K_{0}^{\prime}(\tilde{s} \tilde{r})}{K_{0}(\tilde{s} \tilde{r})} \phi .
$$

Like in our wave equation calculations for $\bar{s}$ large we have

$$
\phi_{\tilde{r}}=-\frac{\left(\tilde{s}+\frac{1}{2 \tilde{r}}+\delta\right) \tilde{s}}{\tilde{s}+\delta} \phi
$$

where

$$
\begin{aligned}
\tilde{r}=\sqrt{\tilde{x}^{2}+\tilde{y}^{2}} & =\sqrt{\frac{x^{2}}{1-M_{\infty}}+y^{2}}=r \sqrt{\frac{\cos ^{2} \theta}{1-M_{\infty}}+\sin ^{2} \theta} \\
& =\frac{r}{\sqrt{1-M_{\infty}}} \sqrt{1-M_{\infty} \sin ^{2} \theta} .
\end{aligned}
$$

Therefore the chain rule yields

$$
\frac{\partial \phi}{\partial \tilde{r}}=\frac{\sqrt{1-M_{\infty}^{2}}}{\sqrt{1-M_{\infty}^{2} \sin ^{2} \theta}} \frac{\partial \phi}{\partial r} .
$$


Note that

$$
\phi=\bar{\psi} e^{-\lambda x}=\bar{\psi} e^{-\lambda r \cos \theta}
$$

Therefore

$$
\frac{\partial \phi}{\partial r}=\left(\frac{\partial \bar{\psi}}{\partial r}-\lambda \cos \theta \bar{\psi}\right) e^{-\lambda r \cos \theta}
$$

Noting

$$
\lambda=\frac{U_{\infty} s}{C_{\infty}^{2}-U_{\infty}^{2}}=\frac{M_{\infty}}{\sqrt{1-M_{\infty}^{2}}} \tilde{s}
$$

and letting $H(\theta)=\frac{\sqrt{1-M_{\infty}^{2}}}{\sqrt{1-M_{\infty}^{2} \sin ^{2}(\theta)}}$ we see that

$$
H(\theta)\left(\frac{\partial \bar{\psi}}{\partial r}-\frac{M_{\infty}}{\sqrt{1-M_{\infty}^{2}}} \tilde{s} \cos \theta \bar{\psi}\right)=-\frac{\left(\tilde{s}+\frac{1}{2 r} H(\theta)+\delta\right)}{\tilde{s}+\delta} \tilde{s} \bar{\psi}
$$

Therefore

$$
\begin{gathered}
\frac{\partial \bar{\psi}}{\partial r}=-\frac{1}{H(\theta)}\left(\frac{\tilde{s}+\frac{1}{2 r} H(\theta)+\delta}{\tilde{s}+\delta}\right) \tilde{s} \bar{\psi}+\frac{M_{\infty}}{\sqrt{1-M_{\infty}^{2}}} \tilde{s} \cos \theta \bar{\psi} \\
\frac{\partial \bar{\psi}}{\partial r}=-\left(\frac{\mathcal{S}(\theta)+\frac{1}{2 r}+\delta \mathcal{S}(\theta)}{\tilde{s}+\delta}\right) \tilde{s} \bar{\psi}
\end{gathered}
$$

where

$$
\mathcal{S}(\theta)=\frac{\sqrt{1-M_{\infty}^{2} \sin ^{2} \theta}-M_{\infty} \cos \theta}{\sqrt{1-M_{\infty}^{2}}}
$$

Upon simplification we propose a boundary operator for the difference of pressure $\psi=p-p_{\infty}$ in the form

$$
\left(\frac{1}{d} \frac{\partial}{\partial t}+\delta\right) \psi_{r}=-\left(\frac{\mathcal{S}(\theta)}{d} \frac{\partial}{\partial t}+\frac{1}{2 R}+\delta \mathcal{S}(\theta)\right) \cdot \frac{1}{d} \psi_{t}-\delta G(\theta, t) \psi
$$

where $d=\sqrt{C_{\infty}^{2}-U_{\infty}^{2}}$. Again one can establish the form of $G$ as

$$
G(\theta, t)=\frac{H(\theta)}{R \ln \left(\frac{2 t H(\theta) d}{R}+D\right)} .
$$

Corrections to $O\left(\frac{1}{R^{3}}\right)$ yield in this case

$$
\delta=\frac{1}{4 R} \frac{\sqrt{1-\bar{M}_{\infty}^{2}}}{\sqrt{1-M_{\infty}^{2} \sin ^{2} \theta}} \frac{1}{1-2 R G} .
$$

In conclusion, this paper points out potential problems in computing solutions of wave-like equations in exterior regions. Remedy of the problems dictates logarithmic correction terms in posing radiation conditions. An extension is proposed to the Euler equations in the far field. 


\section{References}

[1] B. Engquist and A. Majda. Absorbing Boundary Conditions for the Neumerical Simulation of Waves Math. Comp., Vol. 31, (1977), 629-651.

[2] Saul S. Abarbanel et. al. Secondary Frequencies in the Wake of a Circular Cylinder with Vortex Shedding J. Fluid Mech., Vol. 225, (1991), 557-574.

[3] T. M. Hagstrom, S. I. Hariharan and R. C. MacCamy. Asymptotic Behaviors of Absorbing Boundary Conditions, (in preparation)

[4] A. Bayliss and E. Turkel. Radiation Boundary Conditions for Wave-like Equations Comm. Pure Appl. Math., 33, (1980), 707-725.

[5] T. Hagstrom. Consistency and Convergence for Numerical Radiation Conditions, ICOMP Report No. 90-21, NASA TM-103262, (1990).

[6] S.I. Hariharan and R. C. MacCamy. Low Frequency Acoustic and Electromagnetic Scattering, Appl. Num. Math. Vol. 2., (1986), 29-35.

[7] S.I. Hariharan, R.C. MacCamy and C. Muralikrishna. Low Frequency Corrections for the Reduced Wave Equation, to appear.

[8] R.C. MacCamy. Low Frequency Acoustic Oscillations, Quant. Appl. Math., Vol. 23, (1965), 247-256.

[9] S.I. Hariharan. Inverse Scattering for an Exterior Dirichlet Problem, Quant. Appl. Math., No. 2, (1982), 273-286.

[10] L.A. Muravei. On the Asymptotic Behavior for Large Values of Time, of Solutions of Exterior Boundary Value Problems for the Wave Equation with Two Space Variables, Math. USSR Sbornik, Vol. 35, No. 3, (1979), 377-423.

[11] B. Engquist and L. Halpern. Far Field Boundary Conditions for Computation over Long Time, Appl. Numer. Math., Vol. 4, No. 1 (1988), 21-25.

[12] M.E. Goldstein. Unsteady Vortical and Entropic Distortions of Potential Flows Round Arbitrary Obstacles, J. Fluid Mech., Vol. 89, (1978), 433-468. 
[13] H.M. Atassi. Unsteady Vortical Disturbances Around Bodies, Proceedings of the Tenth U.S. National Congress of Applied Mechanics, J.P. Lamb, Ed., ASME, (1986), 475-484.

[14] J.R. Scott and H.M. Atassi. Numerical Solution of Periodic Vortical Flows about a Thin Airfoil, AIAA Paper 89-1691.

[15] Eds. A. Dervieux, Brian Van Leer, J. Periaux and A. Rizzi. Numerical Simulation of Compressible Euler Flows: a GAMM Workshop, Braunschweig: Vieweg (1989).

[16] P. Werner. Low Frequency Asymptotics for the Reduced Wave Equation in Two Dimensional Exterior Spaces, Math. Meth. in Appl. Sci., Vol. 8, (1986) 134-156.

[17] R. Kress. On the Low Wave Asymptotics for the Two-dimensional Exterior Dirichlet Problem for the Reduced Wave Equation, Math. Meth. in Appl. Sci., Vol. 9, (1987), 335-341.

[18] B. Gustafsson. Far Field Boundary Conditions for Time Dependent Hyperbolic Problems, SIAM J. Sci. Stat. Comp., 9, (1988), 812-828. 


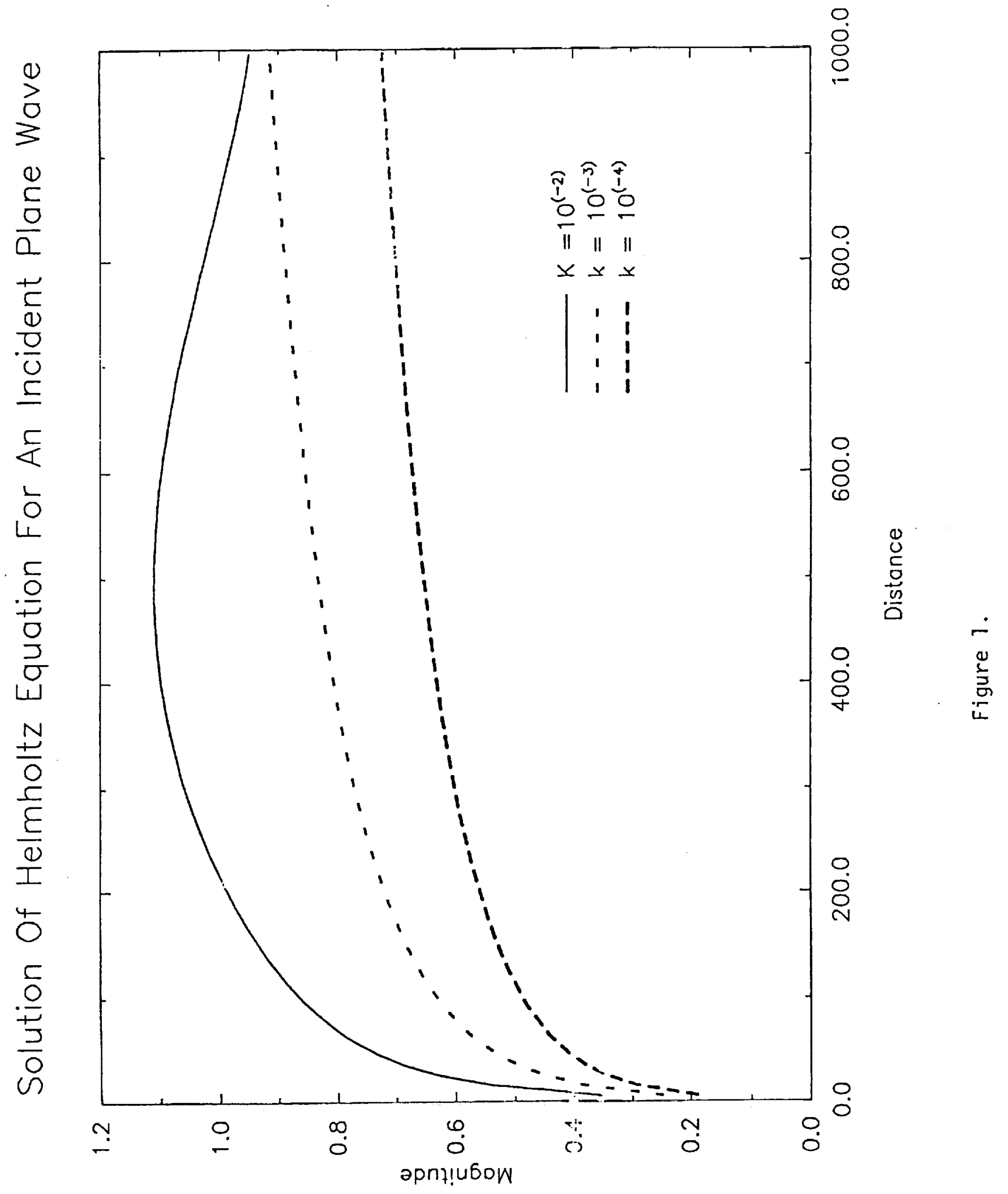




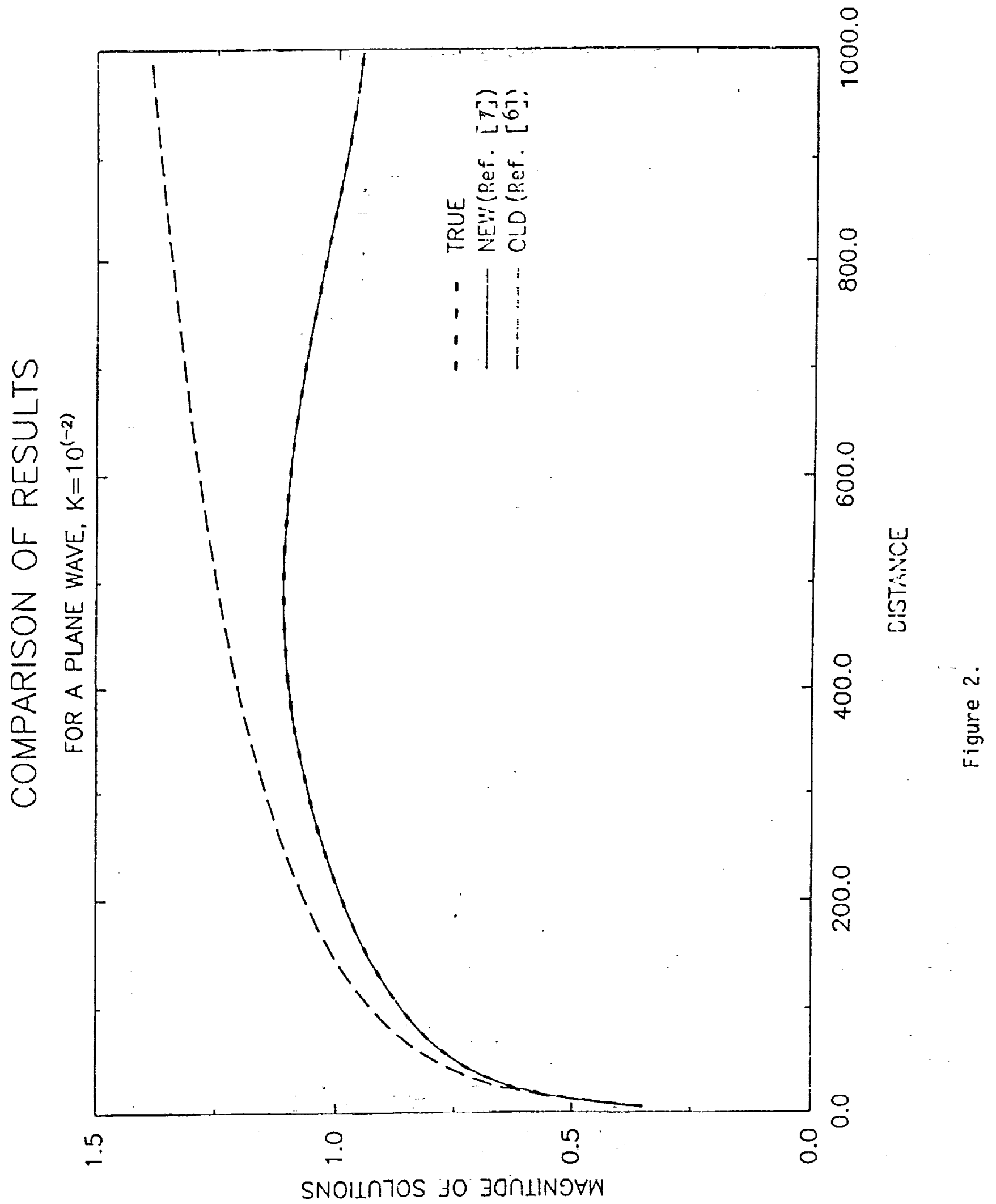




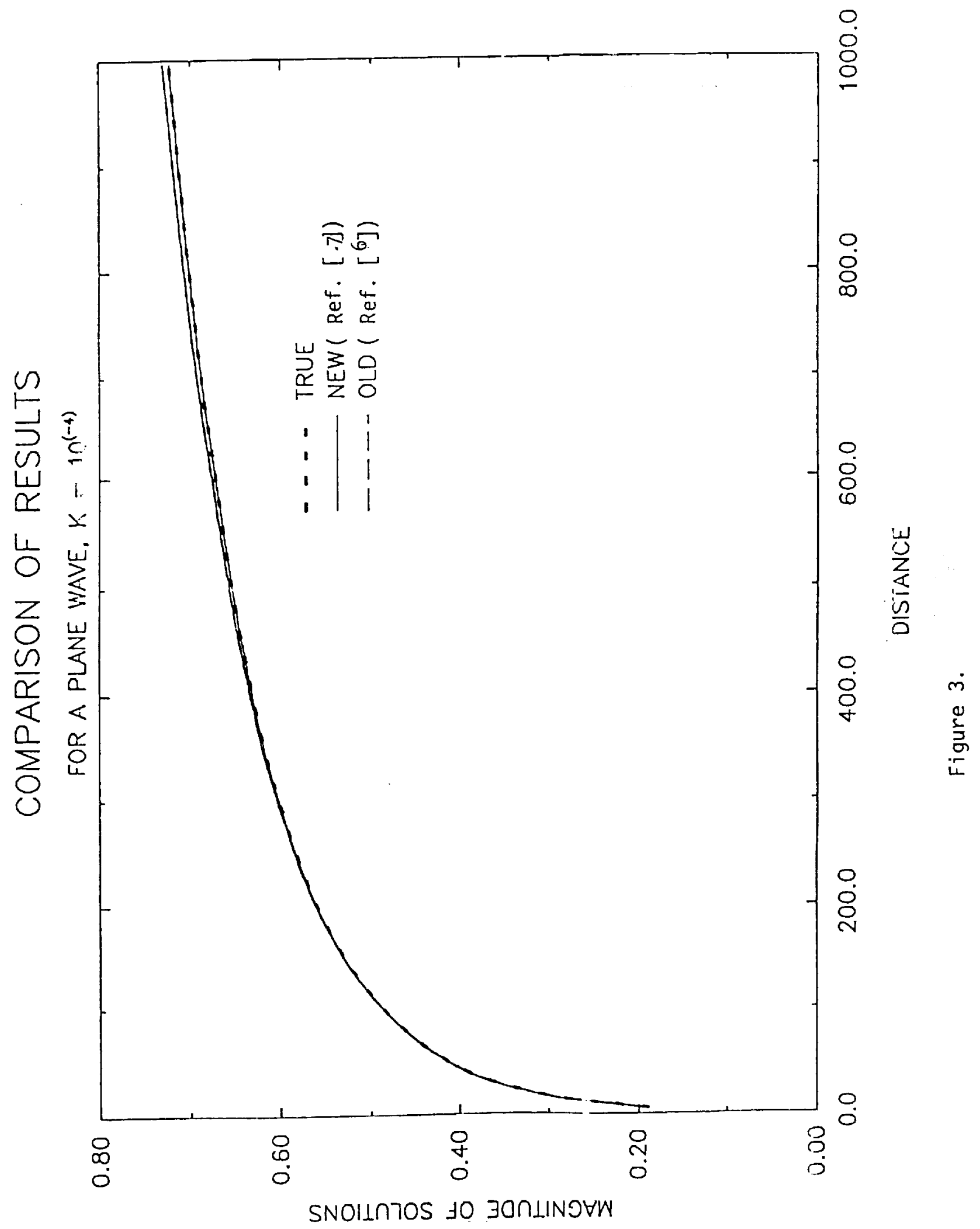




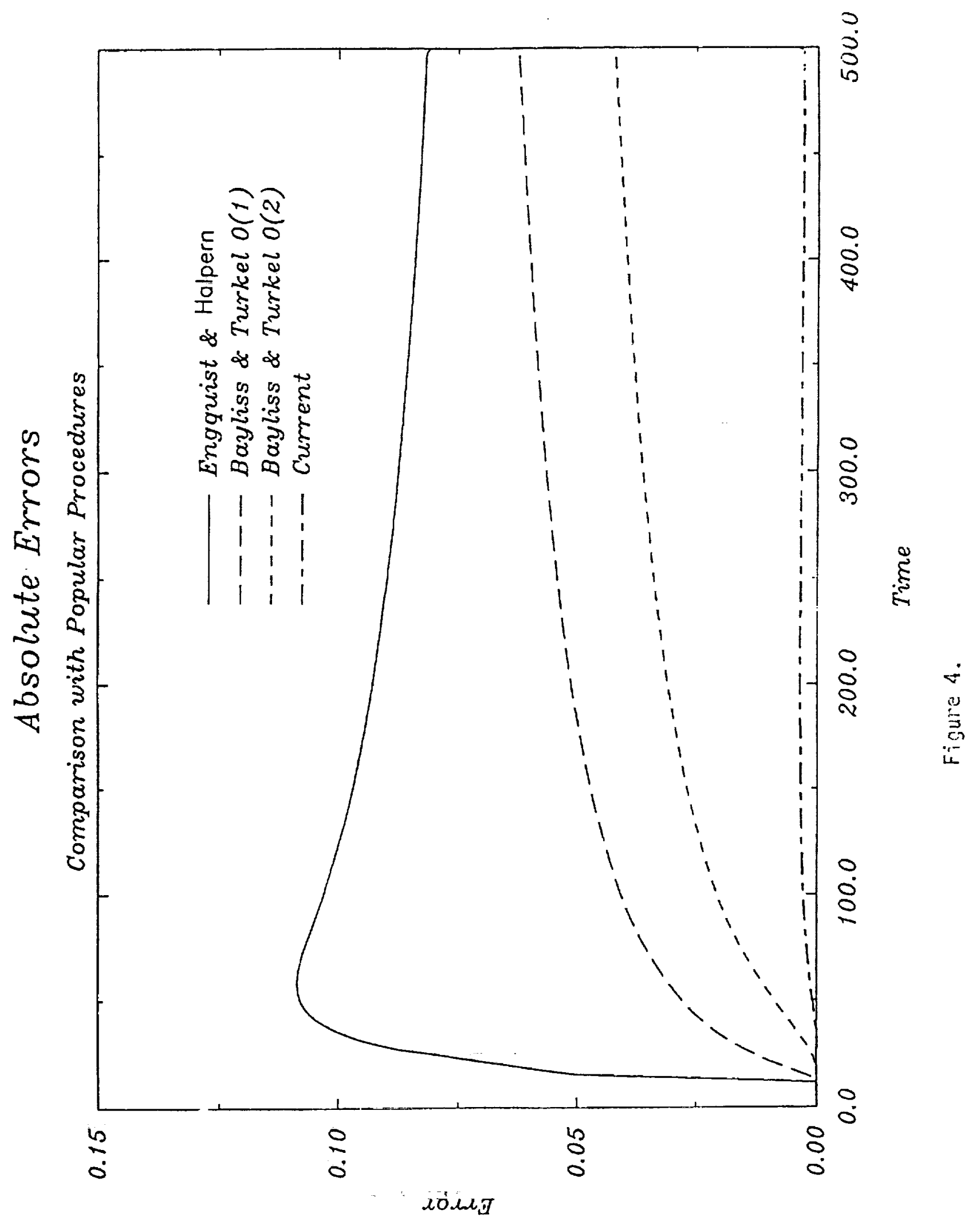




\section{REPORT DOCUMENTATION PAGE}

Public reporting burden for this collection of information is eslimaled to average 1 hour per response, including the time for reviewing instructions, searching existing data sources, gathering and maintaining the data needed, and completing and reviewing the collection of information. Send comments regarding this burden estimate or any other aspect of this collection of information, including suggestions for reducing this burden, to Washington Headquarters Services, Directora Davis Highway, Suite 1204, Arlington, VA 22202-4302, and to the Office of Management and Budget, Paperwork Reduction Project (0704-0188), Washington. DC 20503.

\begin{tabular}{|l|l|l} 
1. AGENCY USE ONLY (Leave blank) & $\begin{array}{c}\text { 2. REPORT DATE } \\
\text { March } 1992\end{array}$ & $\begin{array}{c}\text { 3. REPORT TYPE AND DATES COVERED } \\
\text { Technical Memorandum }\end{array}$
\end{tabular}

4. TITLE AND SUBTITLE

Long Time Behavior of Unsteady Flow Computations
5. FUNDING NUMBERS

WU-505-62-21

8. PERFORMING ORGANIZATION REPORT NUMBER

E-6912

National Aeronautics and Space Administration

Lewis Rescarch Center

Cleveland, Ohio 44135-3191

10. SPONSORING/MONITORING AGENCY REPORT NUMBER

National Aeronautics and Space Administration

Washington, D.C. 20546-0001

NASA TM-105584

ICOMP-92-04

\section{SUPPLEMENTARY NOTES}

S.I. Hariharan, University of Akron, Dept. of Mathematical Sciences, Akron, Ohio 44325 and Institute for Computational Mechanics in Propulsion, Lewis Research Center (work funded under NASA Cooperative Agreement NCC3-233). ICOMP Program Dircctor, Louis A. Povinclli, (216) 433-5818.

12a. DISTRIBUTION/AVAILABILITY STATEMENT

12b. DISTRIBUTION CODE

Unclassified - Unlimited

Subject Category 34

\section{ABSTRACT (Maximum 200 words)}

This paper addresses a specific issue of time accuracy in the calculations of external aerodynamic problems. The class of problems that is discussed here consists of inviscid compressible subsonic flows. These problems are inherently governed by : convective equation. This is readily seen by lincarizing the Euler equation which results in a convective wave equation for the pressure. A key mathematical issue that is not well understood in literature for these problems is the long time behavior of the solution. This is an important aspect if one desires transient calculations of problems governed by the Euler equations or its derivatives such as the small disturbance cquations or the potential formulations of the gust problem. In particular, difficulties arise for two-dimensional problems. In two dimensions the time decay rate of solutions of the wave equation is known to be rather slow. This applies to the convective wave equation as well. The conscquences are rather severe if one focuses on the time accuracy of solutions of problems governed by the Euler equations. In concert with the above mentioned problem, exterior flows require proper modeling of boundary conditions. In particular, computations of these flow problems require truncation of infinite regions into finite regions with the aid of artificial boundaries. On these boundaries one must imposc boundary conditions that are consistent with the physics as well as guarantee consistency with the original problem posed in the unbounded region.

Moreover, these boundary conditions must have accuracy in time as well as space. Some of the well-known procedures do address the issues of spatial accuracy and have remedy for these conditions. Unfortunately, these procedures do not address the time accuracies, which are crucial for the transient problems. Our treatment is discussed in detail and examples are presented to verify the results.

14. SUBJECT TERMS

Compressible flows; Convective wave equation; Boundary conditions 15. NUMBER OF PAGES

$$
\text { Compressible flows; Convective wave equation; Boundary conditions }
$$

17. SECURITY CLASSIFICATION OF REPORT Unclassified
18. SECURITY CLASSIFICATION OF THIS PAGE

Unclassified
19. SECURITY CLASSIFICATION OF ABSTRACT Unclassified 